Rev. Elev. Méd. vét. Pays trop., 1976, 29 (4) : 313-316.

\title{
Portage de Salmonella chez Testudo sulcata tortue terrestre du Sénégal
}

\author{
M. P. DOUTRE (*) et R. BOCHE $\left({ }^{* *}\right)$ \\ (avec la collaboration technique de B. TOURE $\left({ }^{* * *}\right)$ et A. TALL $\left({ }^{* * *}\right)$ )
}

\begin{abstract}
RÉSUMẼ
Une enquête épidémiologique est effectuée sur le portage chronique de Salmonella chez une tortue africaine Testudo sulcata. 30 prélèvements d'excréments ont permis de mettre en évidence 6 sérotypes : $S$. oranienburg, $S$. corvallis, $S$. durban, $S$. llandoff, $S$. bahrenfeld, $S$. adelalde. $S$. bahrenfeld est isolée pour la première fois au Sénégal.

Les possibilités de contamination de l'homme et d'espèces animales domestiques sont envisagées.
\end{abstract}

Ces dernières années, tout particulièrement dans les pays anglo-saxons, des responsables de la santé publique ont attiré l'attention de l'opinion sur le danger que présentent les tortues, terrestres ou aquatiques, en tant qu'agents propagateurs d'entérobactéries pathogènes des genres Salmonella et Arizona.

De nombreux foyers de salmonelloses humaines, touchant le plus souvent les enfants, ont été rapportés à une contamination provoquée par les chéloniens ou l'eau des bassins dans lesquels ces animaux séjournent. La littérature fournit de nombreux exemples $(3,5,12,14,15,16)$. En 1973, FOX, aux Etats-Unis, cite 35 cas de salmonelloses humaines dont l'origine est expliquée par des contacts avec des tortues, S. braenderup, S. litchfield, S. typhimurium sont alors mises en évidence (8). LAMM et collab. estiment que 280000 cas de salmonelloses infantiles sont dus aux tortues (11). En Angleterre, CLEGG faisant l'historique du sujet, dresse la liste de

(*) Chef du Service de Bactériologie du Laboratoire national de l'Elevage et de Recherches vétérinaires (I. S. R. A.), B. P. 2057, Dakar-Hann, Sénégal.

(**) Chef du Service de Bactériologie de l'Institut Pasteur de Dakar.

$(* * *)$ Technicien supérieur et aide-biologiste au Laboratoire national de l'Elevage et de Recherches vétérinaires (I. S. R. A.). différents sérotypes rencontrés, responsables ou non d'affections chez l'homme: $S$. java, $S$. isangi, $S$. newport, $S$. derby, $S$. paratyphi $B$, S. madelia, S. houtens, S. rubislaw (5). A Tanger, HUET isole les souches suivantes : $S$. newport, $S$. canastel, S. paratyphi B, S. brandenburg, S. clifton, S. kibusi, S. richmond et une Arizona (9). En différents lieux, d'autres auteurs effectuent des observations identiques $(4,10$, $13,18,19$ ).

DELAGE a montré que, lorsqu'une tortue s'infeste, elle reste porteuse de germes pendant un temps qui peut atteindre plusieurs années, donc pratiquement le reste de sa vie. L'animal se comporte d'une manière nettement passive vis-à-vis des Salmonella. Il semble n'y avoir aucun mécanisme d'expulsion ou de neutralisation, même pour des germes introduits directement par voie sanguine $(1,2,6)$. La persistance de la présence dans le tube digestif est facilitée par le fait, qu'en captivité tout au moins, bien des chéloniens sont coprophages et même coprophiles (6).

En raison de toutes ces données, désormais bien connues, des mesures législatives ont été édictées aux Etats-Unis. En décembre 1972, une loi est promulguée, elle interdit l'importation de tortues (ou d'aufs de tortues, ces der- 


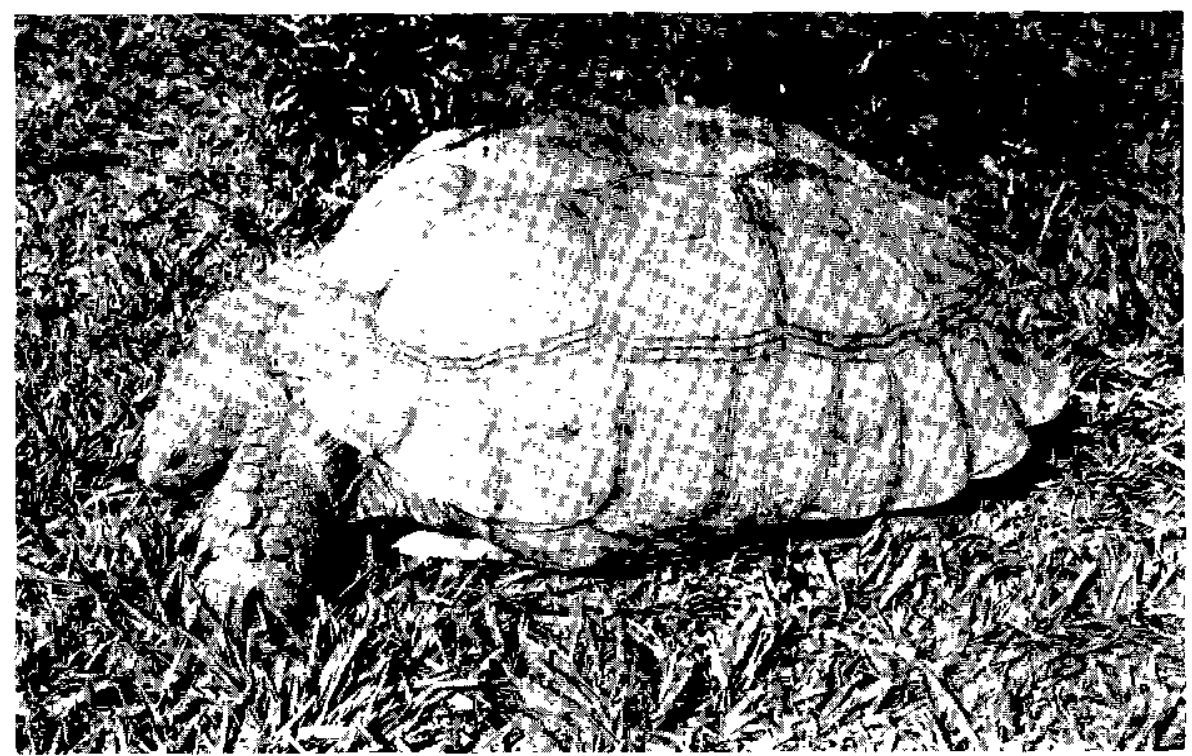

Testudo sulcata (photo D. FRIOT)

niers pouvant aussi héberger des Salmonella (7)) à l'intérieur des frontières, et le transport inter-Etats, pour des sujets qui n'ont pas été certifiés indemnes de Salmonella ou d'Arizona par les autorités du pays expéditeur. Sur le plan bactériologique, des techniques d"isolement plus sensibles sont mises au point (20).

Au Sénégal, une tortue, Testudo sulcata, est souvent conservée dans les cours et les jardins de l'agglomération dakaroise à titre de curiosité (photo). A l'état libre, l'animal vit surtout dans la zone sahélienne et sahélo-soudanienne, fréquentant souvent des régions excessivement arides. Cette espèce, la plus grande des tortues terrestres africaines, atteindrait en 18 ans le poids de $60 \mathrm{~kg}$ pour une longueur de $75 \mathrm{~cm}$, les habitants du sud de la Mauritanie prétendent qu'il existe des individus pesant $150 \mathrm{~kg}$ (17). Dans la nature, Testudo sulcata se nourrit de feuillages divers et semble affectionner particulièrement les feuilles d'Ipomea, mais elle ne refuse pas les herbes les plus sèches. En captivité, l'animal boit assez fréquemment et par quantité appréciable à la fois.

A titre d'information et dans le cadre des enquêtes effectuées sur l'épidémiologie des salmonelloses, des excréments de $T$. sulcata ont été recueillis dans différents jardins (parc zoologique inclus) de l'agglomération dakaroise pour tenter d'apprécier le portage éventuel de Salmonella. La présente publication se propose de présenter les résultats obtenus.

\section{MATÉRIEL ET MÉTHODES}

\section{A. Matériel d'étude}

30 prélèvements coprologiques sent récoltés et transportés en tubes stériles au Laboratoire.

\section{B. Méthodes}

Après enrichissement 48 heures en bouillon sélénite, l'isolement est effectué sur milieu sélectif (désoxycholate citrate lactose).

Comme pour toutes les enquêtes épidémiologiques accomplies jusqu'alors, les souches sont adressées au Centre international des Salmonelles (Institut Pasteur de Paris, Professeur LE MINOR) pour confirmation et détermination des sérotypes.

\section{RÉSULTATS}

6 sérotypes ont été mis en évidence :

$S$. oranienburg (groupe CI) 6,$7 ; \mathrm{m}, \mathrm{t} ;-$

S. corvallis (groupe C3) 8,$20 ; \mathrm{z} 4, \mathrm{z} 23 ;(\mathrm{z6})$

S. durban (groupe DI) 9,$12 ; \mathrm{a} ; \mathrm{e}, \mathrm{n}, \mathrm{z} 15$

S.llandoff (groupe E4) $1,3,19 ; \mathrm{z} 29 ;-$

S. bahrenfeld (groupe H) $6,14,24 ; \mathrm{e}, \mathrm{h} ; \mathrm{I}, 5$

S. adelaide (groupe 0 ) $35 ; \mathrm{f}, \mathrm{g} ;-$

$S$. bahrenfeld est observée pour la première fois au Sénégal, les 5 autres sérotypes ont déjà été rencontrés au moins une fois chez l'homme. 
Il est bien imprudent d'essayer d'établir un taux d'infestation car, au moment de la récolte, on ignore si les excréments appartiennent à un même animal ou à plusieurs. $S$. bahrenfeld est isolée 9 fois de tortues présentes dans un même jardin...

\section{DISCUSSION}

Comme de nombreuses espèces de chéloniens, Testudo sulcata héberge très fréquemment des sérotypes de Salmonella. Dans les lieux d'habitation, l'animal vit souvent en compagnie de volailles, de chèvres, de moutons qui consomment l'eau du même abreuvoir souillé d'excréments de tortues... Des enfants, en jouant, peuvent avoir des contacts avec les pelouses contaminées, les bassins, etc... d'où possibilité d'infection. Le maintien de Testudo sulcata en captivité, non isolée, dans des lieux fréquentés par l'homme ou des espèces animales domestiques présente un danger potentiel lié à la propagation de sérotypes divers de Salmonella, de pouvoir pathogène variable mais pouvant toujours évoluer par passages multiples.

\section{SUMMARY}

Healthy carriers of Salmonella in Testudo sulcata, a tortoise living in Senegal

An epidemiological survey has been carried out in Testudo sulcata, a common african tortoise, to detect healthy carriers of Salmonella. 30 droppings have been studied and have allowed to isolate 6 serotypes : $S$. oranienburg, $S$. corvallis, $S$. durban, $S$. llandoff, $S$. bahrenfeld, $S$. adelaide. $S$. bahrenfeld is demonstrated for the first time in Senegal.

Risks of contamination for men and domestic animals are discussed.

\section{RESUMEN}

\section{Testudo sulcata, tortuga terrestre de Senegal, portadora de Salmonella}

Se efectuó una encuesta epidemiologica sobre Salmonella encontrada de modo cronico en una tortuga africana, Testudo sulcata. Treinta muestras de excrementos permitieron evidenciar seis suerotipos $: S$. Oranienburg, $S$. corvallis, $S$. durban, $S$. llandoff, $S$. bahrenfeld, $S$. adelaide.

Se aisló $S$. bahrenfeld por la primera vez en Senegal.

Se consideran las posibilidades de contaminación del hombre y de especies animales domésticas.

\section{BIBLIOGRAPHIE}

1. BLANC (G.), DELAGE (B.), ASCiONE (L.). Comportement des Salmonella inoculées par voie intracardiaque à Testudo mauritanica. Bull. Soc. Path. exot., 1960, 53 (5) : 131-133.

2. BLANC (G.), DELAGE (B.), ASCIONE (L.). Comportement des Salmonella inoculées par voie sanguine à Testudo graeca. Bull. Soc. Pash. exot., 1960,53 (5) : $774-776$.

3. BORLAND (E. D.). Salmonella infection in dogs, cats, tortoises and terrapins. Vet. Rec., 1975, 96 (18): 401-402.

4. ВОYСОTT (J. A.). Salmonella species in turtles. Science, 1962, 137 : 761.

5. CLEGG (F. C), HEATH (P. J.). Salmonella excretion by terrapins and the associated hazard to human health. Vet. Rec., 1975, 96 (4) : 90-91.

6. DELAGE (B.). Survie des Salmonella ingérées dans le tractus digestif de quelques animaux. Bull. Soc. Path. exot., 1966, 59 (6) : 943-949.

7. FEELEY (J. C.), TREGER (M. D.). Penetration of turtle eggs by Salmonella braenderup. Publ. Health Rep. U.S. A, 1969, 84 (2) : 156-158.

8. FOX (M. D.). Recent trends in salmonellosis epi- demiology. J. am. vet, med. Ass., 1974, 165 (II) : 990-993.

9. HUET (M.). Les Entérobactéries pathogènes isolées à Tanger (1963-1966). Arch. Inst. Pasteur Tunis, 1974, 51 (1-2) : 21-23.

10. KENNEDY (M. E.). Salmonella serotypes isolated from turtle environment. Canad. J. microbiol., 1969, 15 (1) : 130-132.

11. LAMM (S. H.), TAYLOR (A. J.), GANGAROSA (E. J.), ANDERSON (H. W.), YOUNG (W.), CLARK (M. H.), BRUCE (A. R.). Turtle associated Salmonellosis. I. An estimation of the magnitude of the problem in the U. S., 1970-71. Am. J. Epidemiol,, 1972, 95: 511-517.

12. MORSE (E. V.), DUNCAN (M. A.). Salmonellosis an environmentai health problem. $J$. am. vet. med. Ass., 1974, 165 (II) : 1015-1019.

13. ORLANDELLA (V.), Ricerche sui "serbatoi " di Salmonella. Indagini sulla tartaruga (Testudo hermanni $\mathrm{Gm}$ ) sulla lucertola (Lacerta sicula sicula Raf.) e sul riccio (Erinaceus europaeus L.). Nata introduttiva. Atti. Soc. pelorit. Sci. fis. mat. nat., 1967, 13 (3-4) : 193-199. 
14. SABUGO (E. F. P.). Pet turtles in relation to human salmonellosis. Harper Hosp. Bull, 1968, 26 (1) : 31-33.

15. Surveillance of turtle-associated salmonellosis, United States. Morbid and Mortal Rep., 1974, 22; 209 et J. am. vet. med. Ass., 1974, 165 (3) : 255.

16. TOSHKOV (A.), VALERIANOV (T. S.), SLAVKOV(I), KAMBUROV(G.), KARADZHOV (Y.A.), PETKOV (A.), MILANOV (M.), CHILEV (D.), PACHEV (S.), POPOVA (A.), SOKOLOVA (E.). Réservoirs naturels du genre Salmonella chez les tortues dans le district de Burgas (Bulgarie). III. Lysogénie et virulence des souches isolées de tortues. Izvest. mikrobiol. Inst., 1974, 24 : 169-185.
17. VILLIERS (A.). Tortues et crocodiles de l'Afrique noire française (Initiations africaines). 354 p. Dakar, I. F. A. N., 1958.

18. VINCENT (J.), NEEL, LE MINOR (L.). Les Salmonella des tortues. Maroc méd., 1960, 39, 1076.

19. WEBER (A.), PIETZSCH (O.). Ein Beitrag zum Vortommen von Salmonellen bie Landschilkroten aus Zochandlungen und Privathaushalten. Berl. und München. Tierarztl. Woch., 1974, 87. Heft : 13 257-260.

20. WELLS (J. G.), CLARK (G. Mc C.), MORRIS (G. $\mathrm{K}$.). Evaluation of methods for isolating Salmonella and Arizona organisms from pet turtles. Appl. Microbiol., 1974, 27 (1) : 8-10. 